\title{
Depressive Symptom Endorsement among Alzheimer's Disease, Vascular Dementia and Mild Cognitive Impairment ${ }^{*}$
}

\author{
James R. Hall ${ }^{1,2}$, Leigh Johnson ${ }^{2,3}$, April Wiechmann², Robert C. Barber ${ }^{1,4}$, Sid O’Bryant ${ }^{2,3}$ \\ ${ }^{1}$ Institute of Aging and Alzheimer's Disease Research, University of North Texas Health Science Center, Fort Worth, USA \\ ${ }^{2}$ Department of Psychiatry, Behavioral Health and Clinical Neuroscience, University of \\ North Texas Health Sciences Center, Fort Worth, USA \\ ${ }^{3}$ Department of Internal Medicine, University of North Texas Health Science Center, Fort Worth, USA \\ ${ }^{4}$ Department of Pharmacology and Neuroscience, University of North Texas Health Science Center, Fort Worth, USA \\ Email: james.hall@unthsc.edu
}

Received April 8, 2012; revised May 14, 2012; accepted June 10, 2012

\begin{abstract}
Background: The Geriatric Depression Scale (GDS) is widely used to assess depressive symptoms in clinical and research settings. This study utilized a 4 factor solution for the 30-item GDS to explore differences in the presentation of depressive symptoms in various types of cognitive impairment. Method: Retrospective chart review was conducted on 254 consecutive cases of community dwelling elderly newly diagnosed with mild Alzheimer's Dementia (AD) $n=122$, mild Vascular Dementia $(\mathrm{VaD}) \mathrm{n}=71$ or Amnestic Mild Cognitive Impairment (aMCI) $n=32$ and Non-Amnestic MCI $(\mathrm{nMCI}) \mathrm{n}=29$. Results: Analysis revealed no significant differences $(\mathrm{p}<0.05)$ between the groups for total GDS score, the Dysphoria subscale or Cognitive Impairment subscale. AD endorsed significantly fewer symptoms than $\mathrm{VaD}$ on Apathy, Meaninglessness and Dysphoria. AD did not endorse a significantly different number of items than aMCI on any of the subscales. AD endorsed significantly fewer items than nMCI on Apathy and Meaninglessness. VaD endorsed significantly more items than the aMCI only on the Meaninglessness subscale $(p>0.05)$. No statistically significant differences were found between $\mathrm{VaD}$ and $\mathrm{nMCI}$ or between the MCI groups. Conclusions: Support is provided for the use of GDS subscales in a wide range of cognitively impaired elderly. This study suggests in mild dementia the number and type of depressive symptoms vary significantly between $\mathrm{AD}$ and $\mathrm{VaD}$. There are indications that aMCI patients are similar in their symptom endorsement to $\mathrm{AD}$ and $\mathrm{nMCI}$ are similar to $\mathrm{VaD}$ which is consistent with some of the notions regarding likely trajectories of the respective MCI groups.
\end{abstract}

Keywords: Depression; Cognitive Impairment; Alzheimer's; Vascular Dementia; Mild Cognitive Impairment

\section{Introduction}

Depression is a common experience for elderly persons suffering from cognitive impairment and/or dementia. Depression is common in both Alzheimer's disease (AD) and Vascular Dementia (VaD) with research suggesting that $10 \%-20 \%$ of $\mathrm{AD}$ patients suffer from depressive syndromes whereas this estimate climbs to $40 \%$ or more among $\mathrm{VaD}$ patients [1]. Research has shown that for elderly suffering from pre-dementia syndromes depression is one of the most frequently reported neuropsychiatric symptoms found in patients diagnosed with Mild

\footnotetext{
"James R. Hall designed the study, supervised data collection, assisted with data analysis and wrote the manuscript. Leigh Johnson assisted with writing the manuscript. April Wiechmann assisted with writing the manuscript. Robert Barber assisted with writing the manuscript. Sid O'Bryant assisted in the design of the study, assisted with data analysis and assisted with writing the manuscript.
}

Cognitive Impairment (MCI) [2,3]. Elderly patients with MCI and dementia may also exhibit significant depressive symptoms without meeting full diagnostic criteria for a depressive illness $[4,5]$.

Proper identification of depressive symptom themes and affective components of depression may be as important as the clinical diagnosis of depression in understanding the co-occurrence of depression and types of cognitive impairment. Previous research has described symptom themes found in various types of cognitive impairment. In AD patients, symptom clusters with themes of sadness, fatigue, loss of interest, depressed mood, fatigue, and indecisiveness have been identified [6]. Themes among patients with $\mathrm{VaD}$ include depressed mood, loss of interest, no future expectations [7], anhedonia-pessimism, dysphoric tension and cognitive-inhibition [4] While a great deal of research has been done 
on the prevalence of depression in MCI patients [2-11] there is little research that speaks to the specific depressive themes in MCI and their relationship to MCI subtypes [12].

The Geriatric Depression Scale (GDS) was the first screening instrument designed specifically for a geriatric population [13] and is widely used for assessing depressive symptoms in the elderly in both clinical and research settings. The GDS was originally designed and is primarily used as a uni-dimensional instrument to assess depression in the elderly. Most research utilizes conventional cut points for establishing depressed versus nondepressed patient groups and fails to consider the nature of the symptoms endorsed. In demented populations, as with the general population, simple summation of symptoms of depression does not allow for a thorough examination of the importance of particular depressive symptoms and/or symptom clusters and how these may impact functioning. The GDS has been used frequently with patients with cognitive impairment although it has been criticized for not being specifically designed to assess depression in dementia $[14,15]$ and being affected by the stage of dementia [16]. Debruyne et al. [17] argue that the GDS is a reliable screening instrument for patients with MCI but not with AD.

Hall and Davis [18] suggested that the utility of the GDS in screening depression in cognitively impaired populations may be enhanced by using subscales or symptom clusters rather than GDS total score. The factor analysis of the 30-item GDS with cognitively impaired elderly resulted in a four-factor model; 1) Dysphoria, 2) Meaninglessness, 3) Apathy, and 4) Cognitive Impairment. The factor structure found for a cognitively impaired population was noticeably different than that found with a relatively healthy elderly population. This suggests that cognitively impaired elderly express affective experiences differently. Previous research by our group on the relationship of cognition to depression has shown the utility of assessing the four symptom clusters with an older, multi-ethnic rural population [19] as well as individuals diagnosed with MCI [20] and individuals with mild Alzheimer's [21].

The present study examined the prevalence of the GDS-30 symptom clusters among a sample of patients diagnosed with MCI (amnestic and non-amnestic), AD and $\mathrm{VaD}$. Given the prior literature on differential psychiatric manifestations between different dementia pathologies, it was hypothesized that the $\mathrm{AD}$ and $\mathrm{VaD}$ groups as well as the aMCI and nMCI groups would differ significantly in their depressive symptom cluster endorsement. No a priori hypotheses were made regarding possible differences between the MCI and dementia groups.

\section{Method}

\subsection{Participants}

Retrospective review was conducted on 254 consecutive cases of community dwelling elderly (Mean Age $=78.92$ years; Females $=186$, Males $=68$ ) assessed at the UNTHSC Memory Disorders Clinic between January 2009 and December 2011 who were diagnosed with AD, $\mathrm{VaD}$ or MCI and scored 1.0 or less on their Global Clinical Dementia Rating (CDR) score, which is indicative of mild dementia. A consensus diagnosis of $\mathrm{AD}$ or $\mathrm{VaD}$ based on history, physical examination and neuropsychological testing was established. This was based on the criteria of the National Institute of Neurological and Communicative Disorders and Stroke-Alzheimer's Disease and Alzheimer's disease and Related Disorders Association Work Group (NINCDS-ADRDA) [22]. The diagnosis of MCI was established using the criteria of the International Working Group on Mild Cognitive Impairment [23]. Patients meeting criteria for MCI were classified as amnestic (aMCI) if they had a prominent memory impairment, either alone or with other cognitive impairments (multiple domains with amnesia), or nonamnestic (nMCI) if a single non-memory domain was impaired alone or in combination with other non-memory deficits (multiple domains without amnesia).

The final sample consisted of $\mathrm{AD}(\mathrm{n}=122), \mathrm{VaD}(\mathrm{n}=$ 71) aMCI $(n=32)$ and $n M C I ~(n=29)$. The demographic characteristics of the sample are shown on Table 1 . The diagnostic groups differed on age and education with the two dementia groups not differing from each other. Both dementia groups were significantly older and less well educated than the MCI groups. This study was approved by the Institutional Review Board and was performed by means of a retrospective analysis of medical records.

\subsection{Measures}

The GDS 30-item self-rating scale [13] was administered to all participants as part of a neuropsychological assessment. The GDS was administered orally to facilitate patient understanding and increase the clinical utility of the data. The Hall \& Davis [18] four factor solution was used to identify clusters of symptoms. Subscale scores

Table 1. Demographic characteristics of participants.

\begin{tabular}{ccccc}
\hline Demographics & AD & VaD & aMCI & nMCI \\
\hline Male & 30 & 18 & 11 & 9 \\
Female & 92 & 53 & 21 & 20 \\
& & & & 77.26 \\
Age & 80.20 & 80.69 & 76.47 \\
& $(7.265)$ & $(6.295)$ & $(1.101)$ & $(0.879)$ \\
Education & 12.93 & 12.90 & 14.46 & 14.64 \\
& $(31.68)$ & $(3.026)$ & $(2.628)$ & $(2.616)$ \\
\hline
\end{tabular}


were determined by summing the items in each factor producing four scores and the total GDS score was the sum of the thirty items. The methodology and item weightings have been described elsewhere [18]. The Dysphoria subscale consists of eleven items that describe the dysphoric and depressed mood often observed in demented patients. The Meaninglessness factor consists of seven items representing an appraisal of the meaningfulness and purpose (or lack thereof) of one's life with respect to the past, present, and future. The Apathy factor consists of six items representing passive withdrawal from activities and enjoyment of life. The Cognitive Impairment factor consists of six items reflecting affective responses related to decline in cognitive functioning.

\section{Results}

The prevalence of depression in the sample (based on a cut-off score of 10 ) was $18 \%$ for AD patients, 39\% for $\mathrm{VaD}$ patients, $18 \%$ for the aMCI patients and $36 \%$ for the nMCI. ANOVA of total score and subscale scores revealed significant differences between groups on Total GDS score $(p<0.021)$; Apathy $(p<0.012)$ and Meaninglessness $(\mathrm{p}<0.016)$. No significant differences were found between groups on the Dysphoria or Cognitive Impairment scale. Table 2 shows the means and standard deviations for all groups by subscale scores and total GDS score. Analysis of differences between the diagnostic groups using MANCOVA were carried out by subscale scores and total GDS score with gender, education and age serving as co-variants. MANCOVA revealed significant effects for diagnostic groups on Dysphoria $(\mathrm{p}<0.010)$, Apathy $(\mathrm{p}<0.047)$ and Meaninglessness scale $(\mathrm{p}<0.040)$. The effect of diagnosis on Total GDS score approached significance $(\mathrm{p}<0.055)$. There was no effect of diagnosis on the Cognitive Impairment scale. Age or gender did not affect any of the scales or total score. Education was a factor only for the Cognitive Impairment scale. Post-hoc analysis (Table 3) revealed that the $\mathrm{AD}$ group endorsed significantly fewer

Table 2. Means and standard deviation by subscale and diagnosis.

\begin{tabular}{ccccc}
\hline Subscales & AD & VaD & aMCI & nMCI \\
\hline Dysphoria & 2.82 & 3.89 & 3.34 & 3.76 \\
& $(1.288)$ & $(3.927)$ & $(2.647)$ & $(3.471)$ \\
Apathy & 1.31 & 1.90 & 1.46 & 1.95 \\
& $(1.299)$ & $(1.375)$ & $(1.261)$ & $(1.731)$ \\
Meaninglessness & 0.96 & 1.62 & 1.04 & 1.64 \\
Cognitive & $(1.249)$ & $(1.702)$ & $(1.138)$ & $(2.105)$ \\
Impairment & $(1.288)$ & $(1.533)$ & $(1.282)$ & $(1.377)$ \\
& 6.33 & 8.55 & 7.48 & 8.00 \\
Total & $(4.518)$ & $(5.982)$ & $(6.078)$ & $(5.389)$ \\
\hline
\end{tabular}

items than the VaD group on Apathy $(p=0.004)$, Meaninglessness $(p=0.003)$ and Dysphoria $(p=0.025)$. The AD group did not endorse a significantly different number of items than the aMCI group on any of the subscales. The AD group endorsed significantly fewer items than the nMCI group on Apathy $(p=0.025)$ and Meaninglessness $(p=0.048)$. The $\mathrm{VaD}$ group endorsed significantly more items than the aMCI group only on the Meaninglessness subscale $(\mathrm{p}<0.05)$. No differences were found between the $\mathrm{VaD}$ group and the nMCI group. There were no statistically significant differences between the MCI groups.

\section{Discussion}

This study investigated the differences in the expression of clusters of depressive symptoms among four groups of patients with clinically diagnosed cognitive impairment. The prevalence of depression in this study (GDS total score $\geq 10$ ), $18 \%$ in patients with $\mathrm{AD}$ and $39 \%$ in patients with $\mathrm{VaD}$, was very similar to the prevalence rates for depressive disorders found by Castilla-Puentes and Habeych [1] using a large sample and applying ICD-9 diagnostic criteria. They found $18.53 \%$ of $\mathrm{AD}$ patients and $44.14 \%$ of $\mathrm{VaD}$ met criteria for a depressive disorder. This difference in prevalence between $\mathrm{AD}$ and $\mathrm{VaD}$ has been attributed to the lack of insight and difficulties with awareness in AD. Lack of awareness has been negatively correlated with the GDS suggesting that GDS scores should decrease as anosognosia becomes more severe [24].

Studies on the prevalence of depression in MCI have reported rates ranging from $15.7 \%$ [25] to $46.1 \%$ [4] to as high as $63 \%$ [26] depending on the nature of the sample and definition of depression. In our small sample we found $26 \%$ of all MCI patients scored 10 and above on the GDS indicating at least a mild depression. The rates of depression for the two subtypes of MCI (18\% of aMCI

Table 3. Comparison of diagnostic groups by subscale.

\begin{tabular}{ccccc}
\hline $\begin{array}{c}\text { Diagnostic } \\
\text { Group }\end{array}$ & Dysphoria & Apathy & Meaninglessness & $\begin{array}{c}\text { Cog. } \\
\text { Impairment }\end{array}$ \\
\hline $\begin{array}{c}\text { VaD } \\
\text { vs. AD }\end{array}$ & $\mathrm{p}=0.025$ & $\mathrm{p}=0.004$ & $\mathrm{p}=0.003$ & $\mathrm{~ns}$ \\
$\begin{array}{l}\text { VaD } \\
\text { vs. } \mathrm{nMCI}\end{array}$ & $\mathrm{ns}$ & $\mathrm{ns}$ & $\mathrm{ns}$ & $\mathrm{ns}$ \\
$\begin{array}{c}\text { VaD } \\
\text { vs. } \mathrm{aMCI}\end{array}$ & $\mathrm{ns}$ & $\mathrm{ns}$ & $\mathrm{p}=0.05$ & $\mathrm{~ns}$ \\
$\begin{array}{c}\mathrm{AD} \\
\text { vs. } \mathrm{nMCI}\end{array}$ & $\mathrm{ns}$ & $\mathrm{p}=0.025$ & $\mathrm{p}=0.048$ & $\mathrm{~ns}$ \\
$\begin{array}{c}\mathrm{AD} \\
\text { vs. } \mathrm{aMCI}\end{array}$ & $\mathrm{ns}$ & $\mathrm{ns}$ & $\mathrm{ns}$ & $\mathrm{ns}$ \\
$\begin{array}{c}\mathrm{AMCI} \\
\text { vs. } \mathrm{nMCI}\end{array}$ & $\mathrm{ns}$ & $\mathrm{ns}$ & $\mathrm{ns}$ & $\mathrm{ns}$ \\
\hline
\end{tabular}


patients; $36 \%$ of nMCI patients) are almost identical to those of the $\mathrm{AD}$ and $\mathrm{VaD}$ groups respectively. These findings are contradictory to those of Edwards et al. [3] who found that patients with aMCI were more likely to exhibit depressive symptoms than other subtypes of MCI.

The impact of demographic factors on the expression of depressive symptoms and symptoms clusters was found to be minimal. Neither gender nor age was related to different rates of total symptoms or clusters. It is interesting that education was related only to the Cognitive Impairment scale regardless of diagnosis. Individuals with higher education tended to score higher on this subscale that measures "concern with cognitive decline". It may be that individuals with higher education recognize on some level that their current functioning represents a significant change from their previous level of functioning.

The VaD group scored significantly higher than the AD group on dysphoria, apathy and meaninglessness. These findings may reflect the lack of awareness in AD even in the mild stage. The present study is consistent with other evidence that depressed mood, loss of interest and lack of positive expectations of the future are most sensitive in predicting a depressive disorder in $\mathrm{VaD}$ [7]. Individuals with more depressive symptoms are more likely to have a history of vascular events, subclinical vascular disease, and MRI evidence of vascular disease [10]. These findings also may reflect the characteristics of "vascular depression". The subscales give a good description of the nature of the depressive symptoms experienced in $\mathrm{VaD}$ and provide a more complete understanding of the affective experience of patients with mild $\mathrm{VaD}$.

Exploratory post-hoc analysis comparing VaD patients $(n=27)$ who scored in the depressed range and AD patients $(n=22)$ scoring 10 or above revealed no significant differences between the groups on total score, Dysphoria, Cognitive Impairment or Apathy. The VaD group did score significantly higher than the AD group on the Meaninglessness subscale $(p<0.05)$. These findings suggest that as measured by the GDS the presentation of a significant depression in mildly demented individuals is qualitatively similar although depressed $\mathrm{VaD}$ patients may present with more cognitive symptoms of negative self evaluation.

The nMCI group when compared to the AD group shows a pattern similar to that of the $\mathrm{VaD}$ group. The nMCI group scores significantly higher than the AD group on Apathy and Meaninglessness. Apathy has frequently been associated with vascular dementia [27] and advanced Alzheimer's disease [28] and has been found to be present in MCI although apathy is found more frequently in aMCI compared to nMCI [29].

The finding that the nMCI group scored significantly higher than AD on the Meaninglessness subscale may be related to the reduced awareness found in Alzheimer's disease. The Meaninglessness subscale is made up of items that require the person to compare their perception of their current functioning and value with the past. This requires the ability to recognize or "be aware" to make such comparisons. Similarly the $\mathrm{VaD}$ group scored higher than the aMCI group on the meaninglessness subscale. Because of greater cognitive impairment and higher level of awareness, patients with $\mathrm{VaD}$ may recognize the disparity between their past functioning and their current ability.

The scores of the $\mathrm{VaD}$ group were not significantly different from the nMCI on total score or any of the subscales. Similarly the AD group did not differ from the aMCI group on total score or any subscales. The two MCI groups were not significantly different on any scores. The pattern of differences found between the dementia groups and the MCI groups is suggestive of that there are similar patterns of symptom endorsement for $\mathrm{VaD}$ and nMCI groups and similar patterns for the $\mathrm{AD}$ and aMCI groups. This pattern of endorsement is also in line with the literature suggesting that aMCI patients may be more likely to progress to $\mathrm{AD}$ while nMCI patients may be more likely to progress to VaD. Future studies should examine whether or not these symptom endorsement profiles are useful in predicting 1) progression to dementia among MCI patients as well as 2) specific progression from MCI subtype to specific dementia syndrome.

There are limitations affecting the generalizability of the results of this study. The sample is a relatively small clinical sample of convenience rather than a randomly selected community-based sample meaning that the current findings are most applicable to clinical rather than population-based settings. The patients in our sample were also relatively well educated. The patients were referred or sought out a clinic specifically for the diagnosis and treatment of cognitive impairment in the elderly. Nonetheless the results support the use of the GDS factor scores to distinguish characteristics of affective presentation in different forms of cognitive impairment. This information will be helpful in understanding the nature of the affective experience of individuals with cognitive impairment and may prove useful in predicting progression from pre-dementia to dementia syndromes. Future studies should also examine the utility of these symptom clusters in predicting response to or even development of novel therapeutics with cognitively compromised populations.

\section{REFERENCES}

[1] R. C. Castilla-Puentes and M. E. Habeych, "Subtypes of 
Depression among Patients with Alzheimer's Disease and Other Dementias," Alzheimer's \& Dementia, Vol. 6, No. 1, 2010, pp. 63-69. doi:10.1016/j.jalz.2009.04.1232

[2] C. G. Lyketsos, O. Lopez, B. Jones, A. L. Fitzpatrick, J. Breitner and S. DeKosky, "Prevalence of Neuropsychiatric Symptoms in Dementia and Mild Cognitive Impairment: Results from the Cardiovascular Health Study," Journal of the American Medical Association, Vol. 288, No. 12, 2002, pp. 1475-1483. doi:10.1001/jama.288.12.1475

[3] E. R. Edwards, A. P. Spira, D. E. Barnes and K. Yaffe, "Neuropsychiatric Symptoms in Mild Cognitive Impairment: Differences by Subtype and Progression to Dementia," International Journal of Geriatric Psychiatry, Vol. 24, No. 7, 2009, pp. 716-722. doi:10.1002/gps.2187

[4] T. Gabryelewicz, et al., "Prevalence of Major and Minor Depression in Elderly Persons with Mild Cognitive Impairment-MADRS Factor Analysis," International Journal of Geriatric Psychiatry, Vol. 19, No. 2, 2004, pp. 1168-1172. doi:10.1002/gps.1235

[5] K. Lind, A. Edman, I. Karlsson, M. Sjögren and A. Wallin, "Relationship between Depressive Symptomatology and the Subcortical Brain Syndrome in Dementia," International Journal of Geriatric Psychiatry, Vol. 17, No. 8, 2002, pp. 774-778. doi:10.1002/gps.695

[6] E. H. Rubin, L. L. Veiel, D. A. Kinscherf, J. C. Morris and M. Storandt, "Clinically Significant Depressive Symptoms and Very Mild to Mild Dementia of the Alzheimer Type," International Journal of Geriatric Psychiatry, Vol. 16, No. 7, 2001, pp. 694-701. doi:10.1002/gps.408

[7] P. Naarding, et al., "Depression in Vascular Dementia," International Journal of Geriatric Psychiatry, Vol. 18, No. 4, 2003, pp. 325-330. doi:10.1002/gps.831

[8] O. L. Lopez and J. T. Becker, "Treatment of Alzheimer's Disease," Neurology Review, Vol. 35, No. 9, 2002, pp. 850-859.

[9] O. L. Lopez, et al., "Risk Factors for Mild Cognitive Impairment in the Cardiovascular Health Study Cognition Study: Part 2," Archives of Neurology, Vol. 60, No. 10, 2003, pp. 1394-1399. doi:10.1001/archneur.60.10.1394

[10] D. E. Barnes, G. S. Alexopoulos, O. L. Lopez, J. D. Williamson and K. Yaffe, "Depressive Symptoms, Vascular Disease, and Mild Cognitive Impairment," Archives of General Psychiatry, Vol. 63, No. 3, 2006, pp. 273-280. doi:10.1001/archpsyc.63.3.273

[11] M. Crowe, R. Andel, V. Wadley, S. Cook, F. Unverzagt, M. Marsiske and K. Ball, "Subjective Cognitive Function and Decline among Older Adults with Psychometrically Defined Amnestic MCI," International Journal of Geriatric Psychiatry, Vol. 21, No. 12, 2006, pp. 1187-1192. doi:10.1002/gps.1639

[12] L. Rozzini, et al., "Neuropsychiatric Symptoms in Amnestic and Nonamnestic Mildcognitive Impairment," Dementia and Geriatric Cognitive Disorders, Vol. 25, No. 1, 2008, pp. 32-36. doi:10.1159/000111133

[13] J. A. Yesavage, et al., "Development and Validation of a Geriatric Depression Screening Scale: A Preliminary Report," Journal of Psychiatric Research, Vol. 17, No. 1,
1983, pp. 37-49. doi:10.1016/0022-3956(82)90033-4

[14] A. Kørner, et al., "The Geriatric Depression Scale and the Cornell Scale for Depression in Dementia. A Validity Study," Nordic Journal of Psychiatry, Vol. 60, No. 5, 2006, pp. 360-364. doi:10.1080/08039480600937066

[15] R. S. Wilson, S. E. Arnold, T. L. Beck, J. L. Bienias and D. A. Bennett, "Change in Depressive Symptoms during the Prodromal Phase of Alzheimer Disease," Archives of General Psychiatry, Vol. 65, No. 4, 2008, pp. 439-446. doi:10.1001/archpsyc.65.4.439

[16] T. Muller-Thomsen, S. Arlt, U. Mann, R. MaB and S. Ganzer, "Detecting Depression in Alzheimer's Disease: Evaluation of Four Different Scales," Archives of Clinical Neuropsychology, Vol. 20, No. 2, 2005, pp. 271-276. doi:10.1016/j.acn.2004.03.010

[17] H. Debruyne, et al., "Is the Geriatric Depression Scale a Reliable Screening Tool for Depressive Symptoms in Elderly Patients with Cognitive Impairment?" International Journal of Geriatric Psychiatry, Vol. 24, 2009, pp. 556-662. doi:10.1002/gps.2154

[18] J. R. Hall and T. E. Davis, "Factor Structure of the Geriatric Depression Scale with Cognitively Impaired Older Adults," The Clinical Gerontologist, Vol. 33, No. 1, 2010, pp. 39-48. doi:10.1080/07317110903362127

[19] S. O'Bryant, J. Hall, K. Cukrowicz, M. Edwards, L. Johnson, D. Lefforge, M. Jenkins and A. Dentino, "The Differential Impact of Depressive Symptom Clusters on Cognition in a Rural Multi-Ethnic Cohort: A Project Frontier Study," International Journal of Geriatric Psychiatry, Vol. 26, No. 2, 2011, pp. 199-205. doi: $10.1002 /$ gps. 2514

[20] L. A. Johnson, C. Mauer, D. Jahn, M. Song, L. Wyshywaniuk, J. R. Hall, V. Balldin and S. E. O'Bryant, "Cognitive Differences among Depressed and Non-Depressed MCI Participants: A Project Frontier Study," International Journal of Geriatric Psychiatry, 2012, in Press. doi: $10.1002 /$ gps.3835

[21] J. R. Hall, S. E. O’Bryant, L. A. Johnson and R. Barber, "Depressive Symptom Clusters and Neuropsychological Performance in Mild Alzheimer's and Cognitively Intact Elderly," Journal of Depression Research and Treatment, Vol. 2011, 2011, Article ID: 396958, 6 p.

[22] G. McKhann, D. Drachman, M. Folstein, R. Datzman, D. Price and E. M. Stadlan, "Clinical Diagnosis of Alzheimer's Disease: Report of the NINCDS-ADRDA Work Group under the Auspices of Department of Health and Human Services Task Force on Alzheimer's Disease,' Neurology, Vol. 34, No. 7, 1984, pp. 939-944. doi:10.1212/WNL.34.7.939

[23] B. Winblad, et al., "Mild Cognitive Impairment-Beyond Controversies, towards a Consensus: Report of the International Working Group on Mild Cognitive Impairment," Journal of Internal Medicine, Vol. 256, No. 3, 2004, pp. 240-246. doi:10.1111/j.1365-2796.2004.01380.x

[24] Y. Kashiwa, Y. Kitabayashi, J. Narumoto, K. Nakamura, H. Ueda and K. Fukui, “Anosognosia in Alzheimer's Disease: Association with Patient Characteristics, Psychiatric Symptoms and Cognitive Deficits," Psychiatry and Clini- 
cal Neurosciences, Vol. 59, No. 6, 2005, pp. 697-704. doi:10.1111/j.1440-1819.2005.01439.x

[25] D. C. Chan, J. D. Kasper, B. S. Black and P. V. Rabins, "Prevalence and Correlates of Behavioral and Psychiatric Symptoms in Community-Dwelling Elders with Dementia or Mild Cognitive Impairment: The Memory and Medical Care Study," International Journal of Geriatric Psychiatry, Vol. 18, No. 2, 2003, pp. 174-182. doi:10.1002/gps.781

[26] V. Solfrizzi, et al., "Italian Longitudinal Study on Aging Working Group," Dementia and Geriatric Cognitive Disorders, Vol. 24, No. 1, 2007, pp. 55-64. doi:10.1159/000103632

[27] S. Staekenborg, et al., "Behavioural and Psychological
Symptoms in Vascular Dementia; Differences between Small and Large Vessel Disease," Journal of Neurological Neurosurgical Psychiatry, Vol. 81, No. 5, 2010, pp. 547-551. doi:10.1136/jnnp.2009.187500

[28] M. Chan, W. S. Lim and S. Sahadevan, "Stage-Independent and Stage-Specific Phenotypic Differences between Vascular Dementia and Alzheimer's Disease," Dementia and Geriatric Cognitive Disorders, Vol. 26, 2008, pp. 513-521. doi:10.1159/000178755

[29] J. M. Ellison, D. G. Harper, Y. Berlow and L. Zeranski, "Beyond the 'c' in MCI: Noncognitive Symptoms in Amnestic and Non-Amnestic Mild Cognitive Impairment," CNS Spectrums, Vol. 13, No. 1, 2008, pp. 66-72. 